\title{
Tricking a Cancer Cell into Committing Suicide or Apoptosis
}

\author{
Chintamani $^{1}$
}

Received: 22 January 2017 / Accepted: 22 January 2017 / Published online: 28 January 2017

(C) Association of Surgeons of India 2017

Apoptosis in Greek means "falling of the flower petals" and the term is also used to describe programmed cell death [1]. Thousands of years ago, the ancient Indian sages were perhaps aware of this mechanism and the ways of nature, the creation and its destruction. The "creation" or "shrishti" has been compared in ancient Indian scriptures with a spider that creates its own universe (web) with its saliva and then swallows the same at the very end. Thus, it is "poorna", i.e. complete at the beginning and remains "poorna" or complete at the end [Isha Upanishad].

The ancient Indian texts describe life events as proverbial "waves" in the ocean (whole) that rise from and die into the ocean (whole) while the ocean (whole) remains, as does the cosmos. This truly is an amazing scientific way to understand and describe nature. It is well known that approximately three fourths of the universe, human body and a cell are water, thus making "cell" the tiniest cosmic unit. In order, therefore, to understand the human body (and/or the universe), it would be mandatory to understand the human cell and changes that happen in the cell would automatically reflect in the body and vice versa. Like a cell, the human body is programmed to die at a particular time and moment and this mechanism in the life of a cell is called apoptosis. Cell may die due to injury (necrosis) or may commit suicide (i.e apoptosis).

Kerr, Wyllie, and Currie re-discovered this concept of programmed cell death in the seventies and thus provided another and unique mechanism of "active cell death" in addition to the already known concept of "necrosis or passive cellular death" due to factors like ischaemia, infections, toxins, and chemicals

Chintamani

drchintamani7@gmail.com

VMMC, Safdarjang Hospital, New Delhi, India
[2]. Classically, nature uses apoptosis in order to evolve and emerge, like the tadpole losing its tail in order to become a frog. In humans, the mechanism is evident during embryological development when the fetus looses tissue in between the fingers and toes to have clefts and also the natural sloughing out of the endometrium during menstruation. This is continuously happening in the tissues of human body, animals, and plants to keep the subtle balance between proliferation and cell death in nature [3-6].

\section{Apoptosis and Cancer}

The human body has various defence mechanisms to control cell proliferation thus preventing formation of tumours. Apoptosis is one such mechanism that is triggered by a protein called procaspase- 3 which on activation changes to caspase- 3 and that initiates cell death. In cancers, this mechanism is faulty leading to relentless proliferation of cells. Many cancers are refractory and resistant to body's signals for apoptosis and may also be resistant to the effects of certain chemotherapeutic drugs (that act by mimicking apoptosis) leading to uncontrolled proliferation of cancers. Similarly there are certain viruses like human papilloma virus (HPV) that have been implicated into causation of cervical and oral cancers. These viruses act to prevent apoptosis of the cells that have been transformed by them and one such protein that they help to produce is E6 which binds with and inactivates proapoptotic gene $\mathrm{p}-53$. The mutation of $\mathrm{p}-53$ observed in various cancers further substantiates the mechanism behind uncontrolled cell proliferation or cancer. There are other examples like Epstein-Barr virus producing a protein similar to anti-apoptotic gene BcL-2 leading to cell proliferation. Melanoma cells are also known to avoid apoptosis by inhibiting gene encoding Apaf-1 [2-7]. 
The author and his team have studied pro-apoptotic, antiapoptotic and mismatched repair genes (BCL-2 and Bax) for their role as predictors of response to neo-adjuvant chemotherapy in breast cancer patients. It was observed in this study that mutation in pro- and/or anti-apoptotic genes or in the mismatched repair genes leads to chemoresistance that is associated with poor or no response to chemotherapy. It was also found that responders to chemotherapy developed greater chemotoxicity perhaps due to apoptosis initiated simultaneously in tumour tissue and in the rapidly multiplying normal body cells like those in the scalp and bone marrow. Toxicity thus emerged as a reliable predictor of response to neo-adjuvant chemotherapy in cancers by indicating the degree of apoptosis induced or likely to be induced by therapy $[3,4]$.

\section{Can a Cancer Cell Be Tricked into Dying by Using the Mechnism of Apoptosis?}

A landmark research by Paul Hergenrother and his team (Nature Clinical Biology) nearly found a way around this natural biological process that kick starts apoptosis [7]. They found a synthetic molecule that directly activates procaspase-3 to caspase- 3 thus initiating apoptosis in the tumour. Of the nearly 20,000 compounds that were tried, a molecule called "procaspase activating compound number one" or PAC-1 was found to successfully kill many cancer cells. The process depended on the natural levels of procaspase- 3 in the body. The levels being very high in the lungs, it was found to be very effective in lung cancers. So it was postulated that therapy could be tailored to the cancer, the organ and patient's condition by measuring the levels of pro-caspase-3. This selective treatment was expected to minimize collateral damage and toxic effects of chemotherapy [5-7].

Further research into finding pro-apoptotic compounds that can dodge a cancer cell in to dying while minimally or not affecting the normal cells is needed in order to achieve the end result of prompting a cancer cell in to committing suicide. There are many other factors that may also promote apoptosis like anti-oxidants and activities such as yoga, meditation, spiritual therapies, these also need to be explored and studied. The role of spiritual and yogic therapies is already becoming a very useful arm in the management of cancer as a part of integrative approaches in oncology for providing Indian solution to a universal problem [8].

\section{References}

1. Formigli L, Conti A, Lippi D (April 2004) Falling leaves: a survey of the history of apoptosis. Minerva Med 95(2):159-164

2. Kerr JFR, Wyllie AH, Currie AR (1972): Br J Cancer, 26:239-257

3. Chintamani, Jha BP, Bhandari V, Bansal A, Saxena S, Bhatnagar D (2007) The expression of mismatched repair genes and their correlation with clinicopathological parameters and response to neoadjuvant chemotherapy in breast cancer Int Semin Surg Oncol. Feb 14;4:5

4. Chintamani, Singhal V, Singh JP, Lyall A, Saxena S, Bansal A. Is drug-induced toxicity a good predictor of response to neo-adjuvant chemotherapy in patients with breast cancer? a prospective clinical study.

5. Cummings MC, Winterford CM, and Walker NI (1997): Am J Surg Pathol, 21:88-91

6. "Horvitz's Nobel Lecture". Retrieved 2006-12-17 (2006)

7. Rahul Palchaudhuri, Michael J. Lambrecht, Rachel C. Botham, Kathryn C. Partlow, Tjakko J. van Ham, Karson S. Putt, Laurie T. Nguyen, Seok-Ho Kim, Randall T. Peterson, Timothy M. Fan, Paul J. Hergenrother (2015). A small molecule that induces intrinsic pathway apoptosis with unparalleled speed cell reports 13, 2027-2036 December 1

8. Chintamani (2015 Dec) Integrative oncology-finding an Indian solution to universal problems for holistic cancer care!! Indian. J Surg Oncol 6(4):323-324 